\title{
Design of NarrowBand-IoT Oriented Wireless Sensor Network in Urban Smart Parking
}

\author{
https://doi.org/10.3991/ijoe.v13i12.7886 \\ Wei Zhai \\ Xi'an Aeronautical University, Xi'an, China \\ 2587842805@qq.com
}

\begin{abstract}
With the rapid growth of economy, the process of urbanization is picking up speed. The ensuing fast increase in the number of motor vehicles has aggravated the contradiction between the growing parking demand and the limited parking space in urban areas, which impedes the sustainable development of urban traffic. The traditional parking lot, featured by low efficiency and poor automation, fails to satisfy the soaring parking demand. To improve the traffic management across the city, it is necessary to develop an integrated management platform for urban parking lots. In view of the unique advantage of NarrowBand-Internet of Things (NB-IoT), this paper proposes an urban smart parking management platform based on the NB-IoT and wireless sensor network (WSN), aiming to unlock the full potential of urban parking resources. After briefly introducing the NB-IOT wireless communication and license plate recognition (LPR), the author explained the overall architecture and the image processing algorithm of the NB-IoT oriented urban smart parking plan. Then, the proposed plan was contrasted with traditional wireless communication technology. The results prove that the proposed plan outperformed its traditional counterpart. This research will give a great impetus to the development of the IoT although NB-IoT is still in its nascence
\end{abstract}

Keywords-NarrowBand-Internet of Things (NB-IoT), Wireless Sensor Network (WSN), License Plate Recognition (LPR), Smart Parking

\section{$1 \quad$ Introduction}

As the process of urbanization is picking up speed, there has been a drastic increase in the number of motor vehicles, which adds to the difficulty in car parking in urban areas. What is worse, the traffic problem is exacerbated by the phenomenon of disorderly parking [1]. Facing the soaring parking demand, the traditional parking lot falls short in efficiency, safety and management. However, many urban parking spaces are laid idle due to the poor information dissemination [2]. This calls for a smart parking management platform for urban roads and public facilities. To build such a platform, it may help to consider the Internet of things (IoT).

The IoT marks an irreversible trend of development with the progress in wireless communication and the Internet. According to Groupe Speciale Mobile Association 
(GSMA), the connections to the IoT accounted for $2.8 \%$ of the global mobile connections at the end of 2013, with an annual growth rate of nearly $38 \%$ [3]. The International Data Corporation forecasted that the Internet connectivity will reach 30 billion people around the world by 2020, opening up a market worth 1.7 trillion dollars [4].

The various wireless communication techniques in the IoT mainly fall into shortrange communication and Low-power Wide-Area Network (LPWAN). The former covers Zigbee, WiFi, Bluetooth and Z-Wave [5], while the latter includes authorized and unauthorized spectrum wireless communications. Typical examples of authorized spectrum wireless communication are EC-GSM, LTE Cat-m, and NarrowBand-IoT (NB-IoT), and those of unauthorized spectrum wireless communication are LoRa and SigFox. Despite the freezing of NB-IoT specification in June 2016, significant progress of the NB-IoT has been achieved ever since [6]. At present, global operators and manufacturers are competing to enter the NB-IoT market and spread this technology.

The boom of the IoT is accompanied by the proliferation of the concept of smart city. One of the functions of smart city is smart parking, which consists of online inquiry, booking and payment of spare urban parking spaces [7]. The first step to the smart management of urban parkin lies in vehicle identification [8]. The highdefinition cameras on major urban roads may help to identify vehicles in an accurate manner. In other words, the license plate recognition (LPR) method can be adopted for vehicle identification in the smart parking plan [9]. It is better to use the LPR in synergy with the confirmation of spare parking spaces. A viable option is to build a wireless sensor network (WSN) based on the NB-IOT. The network is made up of both the NB-IoT and geomagnetic sensors. The spare parking spaces can be confirmed by by matching the geomagnetic sensors with location information.

In view of the unique advantage of NarrowBand-Internet of Things (NB-IoT), this paper proposes an urban smart parking management platform based on the NB-IoT and wireless sensor network (WSN), aiming to unlock the full potential of urban parking resources. After briefly introducing the NB-IoT wireless communication and license plate recognition (LPR), the author explained the overall architecture and the image processing algorithm of the NB-IoT oriented urban smart parking plan. Then, the proposed plan was contrasted with traditional wireless communication technology. The results prove that the proposed plan outperformed its traditional counterpart. This research will give a great impetus to the development of the IoT although the NB-IoT is still in its nascence.

\section{NB-IoT}

The NB-IoT is an emerging technology in the field of the IoT. It is also known as Low Power Wide Area Network (WAN), as it consumes low power and enables cellular data connections in wide area networks. Built on the cellular network, the technology consumes only $180 \mathrm{kHz}$ of bandwidth. In addition, the NB-IoT can be deployed at a low cost, because it is directly applicable to Global System for Mobile communications (GSM) and the Universal Mobile Telecommunications System (UMTS) [10]. Compared with Bluetooth, ZigBee and other short range communica- 
tion techniques, mobile cellular networks feature a wide coverage and mobile connectivity, allowing for diverse and colourful applications. Suffice is opening up an unprecedentedly vast market [11]. The business distribution of the IoT is shown in Figure 1.

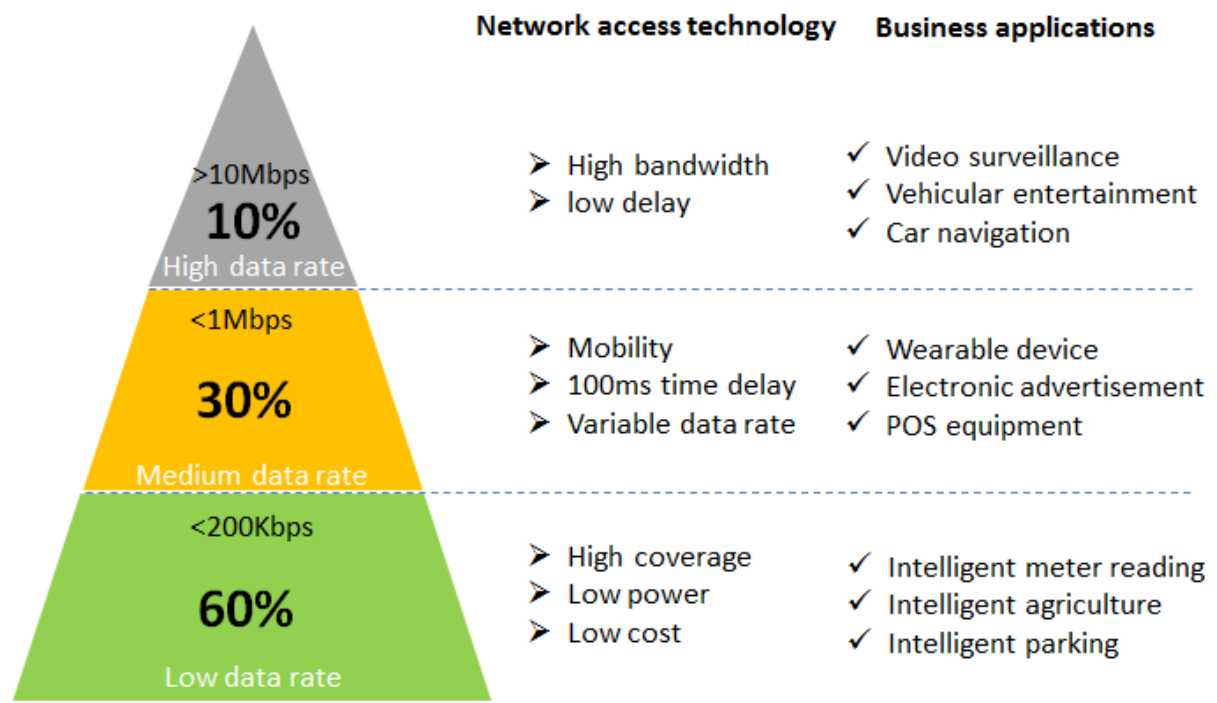

Fig. 1. Business distribution of the IoT

The NB-IoT maintains an edge over other wireless communication methods in the following aspects:

1. Wide coverage: The NB-IoT is an effective guarantee to indoor coverage. At the same frequency band, the NB-IoT gains $20 \mathrm{~dB}$ more than the traditional network, leading to a 100 -fold increase in coverage area. The coverage is both wide and deep, making it possible to apply the technology in rural areas, factories, underground garages, manhole covers, and the like. For example, the NB-IoT does not require the fragile antenna in the GPRS method to monitor manhole covers.

2. Strong connection: In the same base station, the NB-IoT can provide 50-100 times more access than the traditional wireless technology. Taking a smart home as an example, the NB-IoT can easily realize the connections between various devices in the future networking of smart home.

3. Low power consumption: Low power consumption is an important indicator of the application of the IoT. Since the sensors are often located in remote areas, the batteries should not be replaced frequently. The stringent requirement on battery life can be satisfied by the NB-IoT, which minimizes power consumption by developing applications of small data volume and rate.

4. Low cost: The low cost of the NB-IoT is mainly reflected in the reuse of base station. Unlike LoRa, the NB-IoT does not need to re-establish the communication 
network, and multiplexes base stations and antennas. The low data rate, low power consumption and low bandwidth all contribute to the advantage of low cost.

Thanks to the above advantages, it is easy to implement the NB-IoT in various cases, such as remote meter reading, smart parking and smart farming.

\section{LPR}

The LPR is a technology to detect vehicles on the roads and auto-extract license plate information like characters, figures and colours. As an essential part of modern smart traffic system, the LPR has been implemented in vehicle monitoring and public parking. The technology is grounded on digital image processing, pattern recognition and computer vision [12]. The number of each vehicle is acquired by analysing the vehicle images or video sequences taken by the camera. So far, the LPR has been applied to toll collection, parking management, traffic guidance, vehicle scheduling, vehicle detection, etc.

There are many LPR algorithms, and new ones keep emerging. However, most algorithms are designed for stringent environments, namely fixed shooting location, focal length and illumination. In these cases, some of the license plates cannot be recognized.

The LPR system usually consists of the hardware module (data acquisition) and the software module (LPR) [13]. The data acquisition module collects the image of the license plate, and transfers the collected image to the software module [14]. Based on the relevant algorithms, the software module extracts the license plate from the original image, and acquires the information on the license plate.

The flow chart of the LPR technology is as shown in Figure 2.

1. Image acquisition module: Before the image acquisition, it is necessary to install the image acquisition device in the corresponding location. The device usually operates on an autofocus-enabled integrated camera. To adapt to different lighting conditions, the camera should have light compensation function for shooting at different lighting levels. The accuracy of information extraction hinges on the clarity of the captured image.

2. Image identification module: There are different ways to recognize the license plate information from the image in different identification devices. The recognition often contains the four steps below.

(a) Image pre-processing: The images captured in different environments are subject to varied degrees of interference, e.g. weather and lighting. For instance, the plate may be located erroneously in an image due to the noise in the image and over exposure. For accurate recognition of license plate information, the image must be pre-processed to facilitate subsequent image processing. The pre-processing measures include de-noising, morphological processing and binary processing. 


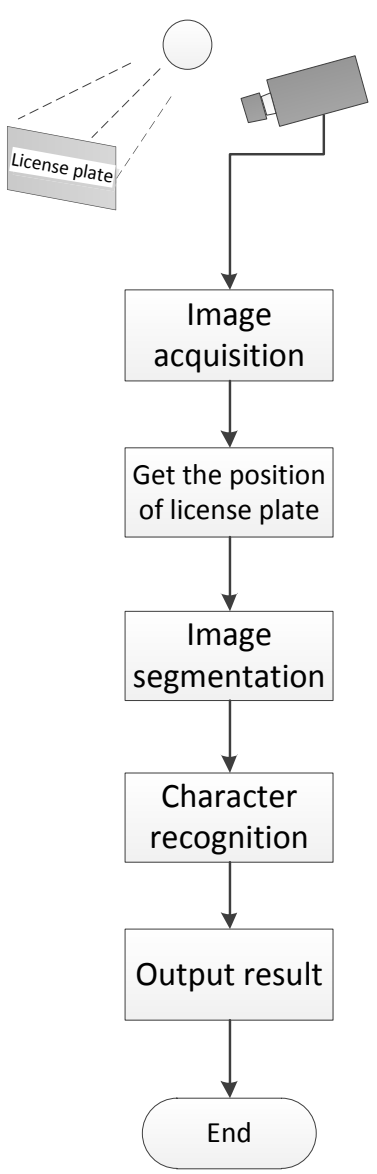

Fig. 2. Flow chart of the LPR

(b) License plate positioning: After the pre-processing process, it is necessary to pinpoint the specific position of license plate in the image, and separate the license plate image from the rest of the image. This step, the key and difficult point in the identification process, has a direct impact on the LPR process.

(c) Character segmentation: In this step, the characters are recognized in the license plate. There are usually many characters in a license plate. However, the attributes of characters cannot be determined before processing. For the ease of character recognition, the specific characters must be segmented from the license plate.

(d) Character recognition: The characters in the license plate are composed of figures and special symbols. These characters are all of the same format and the same size. In general, it is more difficult to identify special symbols than figures. The difficulty is attributable to the similarity of Chinese characters and the wear pattern of the license plate. 


\section{Design of urban smart parking platform based on NB-IoT}

\subsection{Overall architecture}

The hardware part of urban smart parking management platform based on NB-IOT (hereinafter referred to as the proposed platform) mainly includes a parking space detector, an automatic LPR device, a parking guide and a WSN. The software part of the system contains parking area query, mobile payment, smart car search, smart mobile management and smart parking management. In the proposed platform, geomagnetism is employed to detect the parking spaces, the personal digital adaptor (PDA)/mobile app serves as the management tool, and the NB-IoT is taken as the data transmission channel. The PDA and the mobile app are relied on to implement parking management. The overall architecture of the proposed platform is illustrated in Figure 3.

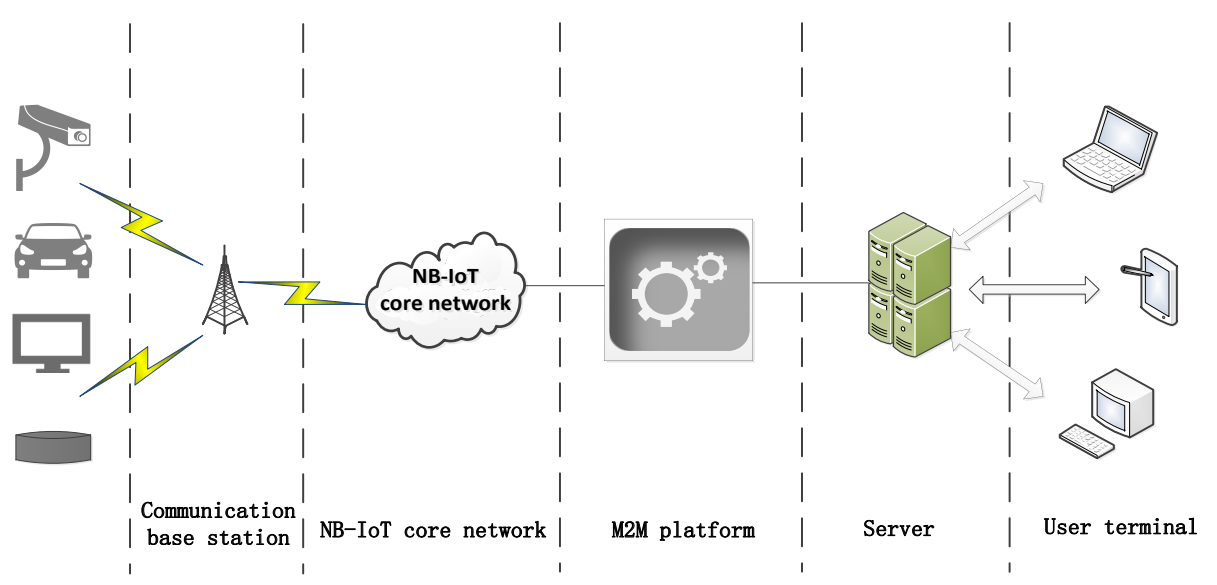

Fig. 3. The overall architecture of the proposed platform

Specifically, the automatic LRP device is used to capture images from the video stream and determine the license plate information through pattern recognition. The vehicle location in the parking lot is acquired by geomagnetic sensors. Whereas LoRa does not support precise monitoring, the location information of each sensor is recorded in the database of the proposed platform. Upon receiving the identity of a sensor, the proposed platform will find out the corresponding location information from the database, thus leading the vehicle to the parking space. The parking space detector is mainly designed to check the occupancy of parking spaces. Once it detects a free parking space, the detector will send the information to the proposed platform, and then guides the vehicle to the proper parking space.

The functions of spare parking query, smart car search and mobile payment are all carried out in the mobile app. Users can find their locations and nearby parking information on the app, which can guide them to the proper parking space. The parking 
fee can be paid automatically if users enter the number of parking spaces in the app. In addition, users can find their own cars with the function of smart car search.

\subsection{Image recognition algorithm}

The BP neural network is a hierarchical neural network model, including an input layer, an output layer and a hidden layer. The three layers are fully connected to each other in a fixed pattern. Of course, the model has only inter-layer connections but no intra-layer connections. The information can be transferred forward or backward in the model. In forward transfer, the input layer is responsible for inputting the information, the hidden layer is responsible for processing the information, and the result is eventually passed to the output layer. The result will be outputted if it lives up to the expectation. Otherwise, the reverse transfer will be conducted until the output is in line with the expectation. The model of BP neural network can be seen in Figure 4.

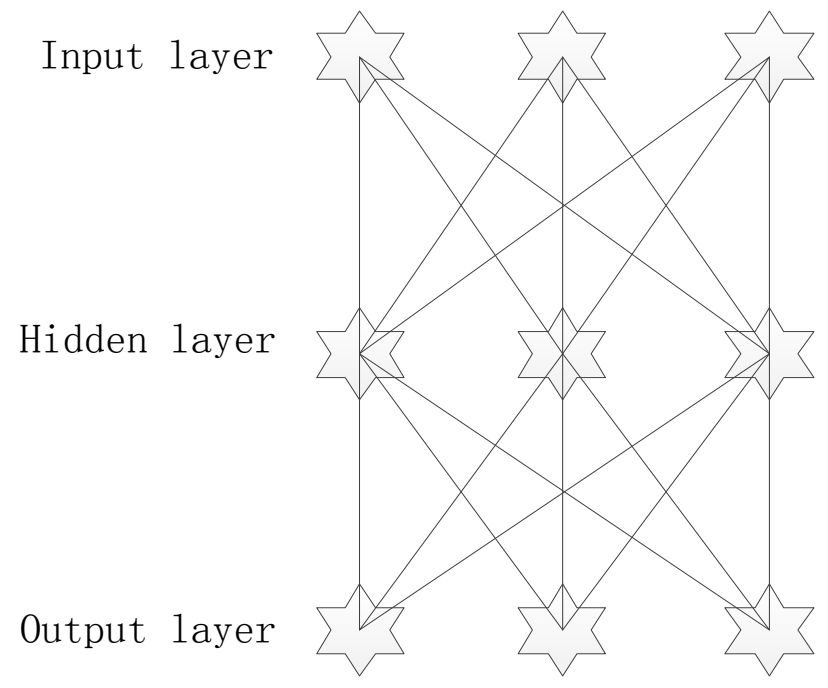

Fig. 4. BP neural network model

Forward transfer model. Let us denote the input vector and weight vector of a neuron as $I$ and $W$, respectively.

$$
\begin{gathered}
I=\left(i_{1}, i_{2}, i_{3} \mathrm{~L} i_{n}\right)^{T} \\
W=\left(w_{1}, w_{2}, w_{3} \mathrm{~L} w_{n}\right)^{T}
\end{gathered}
$$

Let $\varnothing$ be the threshold for neurons. Then, the output of neurons can be obtained by the following formula.

$$
O=f\left(\sum W I-\phi\right)
$$


For the three-level BP neural network, it is assumed that the output vector of the hidden layer is $O_{h}$.

$$
O_{h}=\left(o_{1}, o_{2}, o_{3} \mathrm{~L} o_{m}\right)^{T}
$$

Suppose the weight of the input layer to the hidden layer is $W_{h}$, while the weight of the hidden layer to the output layer is $W_{o}$.

$$
\begin{aligned}
& W_{h}=\left(w_{h 1}, w_{h 2}, w_{h 3} \mathrm{~L} w_{h n}\right) \\
& W_{o}=\left(w_{o 1}, w_{o 2}, w_{o 3} \mathrm{~L} w_{o m}\right)
\end{aligned}
$$

According to initial formula of the activation function, the following equation holds.

$$
f(x)=\frac{e^{x}}{1+e^{x}}
$$

Then, the activation value of a neural unit in the hidden layer can be obtained by the following formula.

$$
h_{j}=\sum_{i=1}^{n}\left(w_{h i} i_{i}-\phi\right)
$$

Finally, the output function of hidden layer can be acquired by substituting the activation value into the activation function.

$$
O_{h}=f\left(h_{j}\right)=\frac{1}{1+\exp \left(-h_{j}\right)}
$$

The activation and output values of the output layer can be obtained in a similar way.

Reverse transfer model. Let us denote the output of the $l$-th neuron in the output layer as $Z_{o l}$. If there is a big error between the actual output and the expected output, it is necessary to carry out the reverse transfer. The error of the output layer is calculated as follows.

$$
d_{o l}=\left(e_{l}-z_{l}\right) z_{l}\left(1-z_{l}\right)
$$

The alignment error of each unit in the hidden layer is as follows.

$$
d_{h l}=\left(\sum_{l=1}^{m} w_{o l} \cdot d_{o l}\right) \cdot z_{l} \cdot\left(1-z_{l}\right)
$$

The corrected weights and thresholds between the output layer and the hidden layer are obtained as: 


$$
\begin{gathered}
\Delta w_{l j}=\alpha \cdot d_{l} \cdot o_{j} \\
\Delta \theta_{l}=\alpha \cdot d_{l}
\end{gathered}
$$

Where $\alpha$ is the learning coefficient $(\alpha>0)$.

Similarly, it is possible to obtain the corrected weights and thresholds between the output layer and the hidden layer. The minimal error of the neural network and the optimal result can be acquired through repetition of the above processes. The flow chart of the LPR based on BP neural network is presented below.

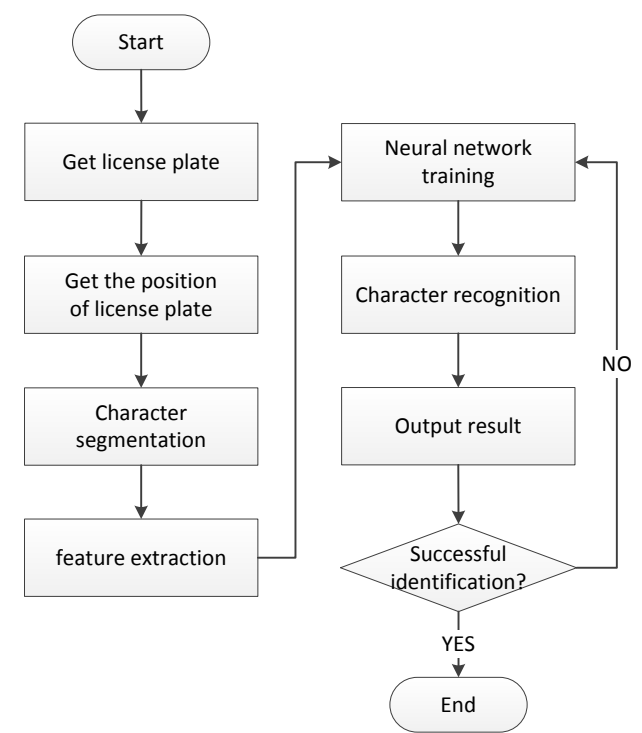

Fig. 5. Flow chart of the LPR based on BP neural network

\section{$5 \quad$ Simulation and analysis}

The power consumption of NB-IoT was compared with that of Zigbee to verify the performance of the proposed platform. The comparison was made in the same environment. Table 1 records the power consumptions of the two techniques.

Table 1. The comparison of energy consumption

\begin{tabular}{|l|c|c|}
\hline \multicolumn{1}{|c|}{ Time } & NB-IoT & Zigbee \\
\hline 1 month & $99 \%$ & $95 \%$ \\
\hline 3 months & $97 \%$ & $89 \%$ \\
\hline 5 months & $95 \%$ & $82 \%$ \\
\hline 7 months & $92 \%$ & $74 \%$ \\
\hline 9 months & $89 \%$ & $65 \%$ \\
\hline 12 months & $85 \%$ & $54 \%$ \\
\hline
\end{tabular}


It can be seen that the NB-IoT consumed less energy than Zigbee, indicating that the technique is cheaper to maintain. In spite of the higher hardware cost, the NB-IoT is more cost efficient than Zigbee, considering the long-term maintenance cost.

\section{Conclusions}

The rapid development of wireless communication technology has created ideal conditions for the construction of smart cities. One of the most important aspects of smart city is smart parking. To improve the traffic management across the city, it is necessary to develop an integrated management platform for urban parking lots. In view of the unique advantage of NarrowBand-Internet of Things (NB-IoT), this paper proposes an urban smart parking management platform based on the NB-IoT and wireless sensor network (WSN), aiming to unlock the full potential of urban parking resources. After briefly introducing the NB-IOT wireless communication and license plate recognition (LPR), the author explained the overall architecture and the image processing algorithm of the NB-IoT oriented urban smart parking plan. Then, the proposed plan was contrasted with traditional wireless communication technology. The results prove that the proposed plan outperformed its traditional counterpart. This research will give a great impetus to the development of the IoT although NB-IoT is still in its nascence.

\section{$7 \quad$ References}

[1] Dai G.H., Yu J.H. (2016). Research on NB-Io T Background, Standard Development, Characteristics and the Service, Mobile Communications, 7, pp. 31-33.

[2] Liu W., Dong J.B., Liu N., Chen Y.L., Han Y.B., Ren Y.B. (2016). NB-Io T key technology and design simulation method, Telecommunications Science, 11, pp. 144-146

[3] Zou Y.L., Ding X.J., Wang Q.Q. (2017). Key Technologies and Application Prospect for NB-IoT, ZTE Technology Journal, 1, pp. 43-45.

[4] Cai C.G. (2017). Analysis of current situation and development trend of NB-IoT low rate and narrowband Internet of things communication technology, Information \& Communications, 3(171), pp. 237-240.

[5] Mangalvedhe N., Ratasuk R., Ghosh A. (2016). NB-IoT Deployment Study for Low Power Wide Area Cellular IoT, IEEE Annual International Symposium on Personal, Indoor, and Mobile Radio Communications. USA, pp. 12-21.

[6] Ratasuk R., Vejlgaard B., Mangalvedhe N. (2016). NB-Io T System for M2M Communication, IEEE Wireless Communications and NETWORKING Conference Workshops. USA: IEEE, pp. 428-432.

[7] Carlos A, Trasvina M., Ruben B. (2016). A Network Performance Analysis of LoRa Modulation for LPWAN Sensor Devices, Ubiquitous Computing and Ambient Intelligence. Germany: Springer, pp. 174-181.

[8] Steyensnavarro E., Wong V., Lin Y.A. (2007). Vertical Handoff Decision Algorithm for Heterogeneous Wireless Networks, IEEE Transactions on Vehicular Technology, 2007, 57(2), pp. 3199-3204.

[9] Lu B. (2016). Analysis on NB-PDCCH Resource Scheduling in NB-IoT, Mobile Communications, 23, pp. 17-19. 
[10] Wang J.Y., Wang X.Z., Wu Q. (2017). Core network service model and networking scheme oriented NB-IoT, Telecommunications Science, 4, pp. 149-151.

[11] Pan S., Tao S., Chen Y.Q. (2017). An Optimal Selection Method of Multi-Cellular Networks Based on NB-Io T Nodes, ZTE Technology Journal, 1(23), pp. 25-27

[12] Su Y.P. (2016). Research and Implementation of license Plate Recognition Technology Based on Image Processing, pp. 9-19.

[13] Ding W. (2011). Application of License Plate Recognition Based on Improved Neural Network, Computer Simulation, 8(28), pp. 359-370.

[14] Sun D.W., Zhu C.H. (2008). Skew Angle Detection of the Vehicle License Plate Image and Correct Based on Radon, Transformation, Microcomputer Applications, 2(29), pp. 1821.

\section{Author}

Wei Zhai is with Xi'an Aeronautical University of China, Xi'an 710077, she is manly engaged in the research of electronics and communication.

Article submitted 25 October 2017. Published as resubmitted by the author 24 November 2017. 